\title{
Evaluación formal de blogs con contenidos académicos y de investigación en el área de documentación
}

\author{
Por Sonia Jiménez Hidalgo y Javier Salvador Bruna
}

\begin{abstract}
Resumen: Los blogs, weblogs o bitácoras son herramientas que permiten la edición de cualquier tipo de contenidos en internet de manera sencilla y rápida. Estas virtudes han hecho que también sean utilizados por la comunidad investigadora y académica para el intercambio de ideas, convirtiéndose, de esta forma, en un nuevo canal que la ciencia y el conocimiento académico dispone para la difusión y confrontación de sus avances. No obstante, y como sucede en otros medios de comunicación científica más tradicionales, parece necesario y positivo el establecimiento de una serie de criterios de calidad que permita determinar en qué medida dichos medios son una forma fiable de intercambio de información. En este artículo se analizan aquellos aspectos de la calidad relacionados con la estructura formal de los blogs que incorporan contenidos de carácter científico y académico, sin tener en cuenta, por el momento, sus contenidos.
\end{abstract}

Palabras clave: Blogs científicos, Blogs académicos, Software social, Herramientas colaborativas, Evaluación de la calidad formal.

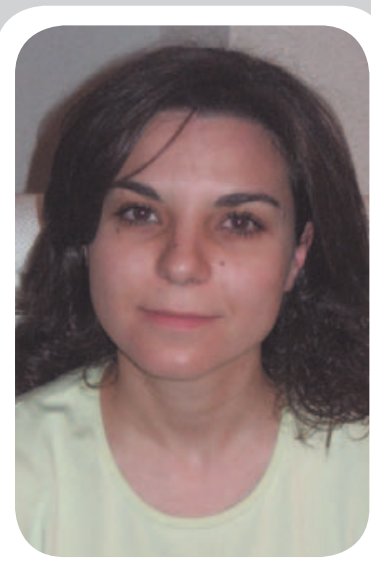

Sonia Jiménez Hidalgo, licenciada en biología, trabaja como colaborador de $I+D+l$ en el Cindoc del CSIC y es asesor técnico editorial de la Revista española de documentación científica.

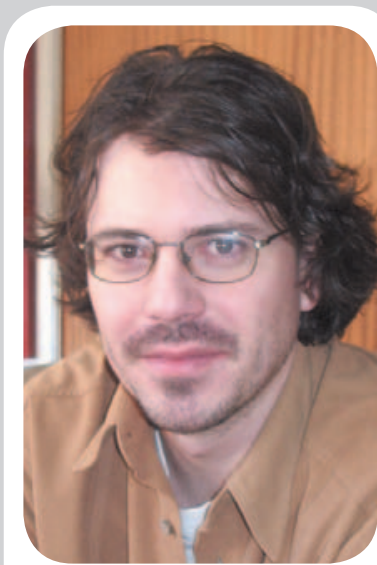

Javier Salvador Bruna, licenciado en documentación, es colaborador de $1+D+l$ en el Departamento de Innovación Tecnológica en Información Científica del Cindoc del CSIC.

\section{Title: Formal assessment of academic and scientific blogs on information science}

Abstract: Weblogs (or blogs) are tools for editing and publishing all types of content on the Internet, easily and quickly. The research and academic community has taken advantage of them for exchanging ideas and information, thus converting blogs into a new communication channel to disseminate and discuss new scientific advances. However, as has occurred with other traditional means of scientific communication, it would be useful to establish quality criteria for determining the extent to which blogs are a reliable way to exchange scientific ideas. In this article the quality of the formal structure of the blogs is analysed, as opposed to the quality of their content.

Keywords: Scientific blogs, Academic blogs, Social software, Collaborative tools, Formal quality assessment.

Jiménez Hidalgo, Sonia; Salvador Bruna, Javier. "Evaluación formal de blogs con contenidos académicos y de investigación en el área de documentación”. En: El profesional de la información, 2007, marzo-abril, v. 16, n. 2, pp. 114-122.

DOI: 10.3145/epi.2007.mar.03

\section{Introducción}

El desarrollo de internet ha supuesto importantes cambios en los esquemas de comunicación de la ciencia. Asimismo, la web está experimentando hoy día una nueva transformación. Estamos evolucionando de una de naturaleza estática, empleada básicamente para localizar información y sin demasiada interactividad, a un nuevo espacio cuyo núcleo fundamental es la colaboración. Berners-Lee la ideó como un espacio colaborativo de intercambio de información con sus colegas del CERN. Con la aparición de la web 2.0, de algún modo, estamos volviendo a asumir algunos de los principios que fueron fundamentales en sus oríge- nes, lo que supone una nueva oportunidad de fomentar y potenciar la transmisión de conocimiento, así como de darle visibilidad al mismo.

Es cierto que existen muchas reticencias relacionadas con el cambio de los modelos de comunicación científica tradicional hacia estos nuevos sistemas. Sin embargo, la aparición de herramientas como los weblogs (o blogs) y los wikis supone que el fin último del trabajo del investigador, es decir, la comunicación de sus trabajos y resultados de investigación a sus colegas de todo el mundo, se pueda realizar de una manera rápida. Además, no hay que olvidar que igualmente lo será la respuesta de los miembros de la comunidad 


\section{baratz}

\section{En primera línea en}

Sistemas de Información y Gestión del Conocimiento
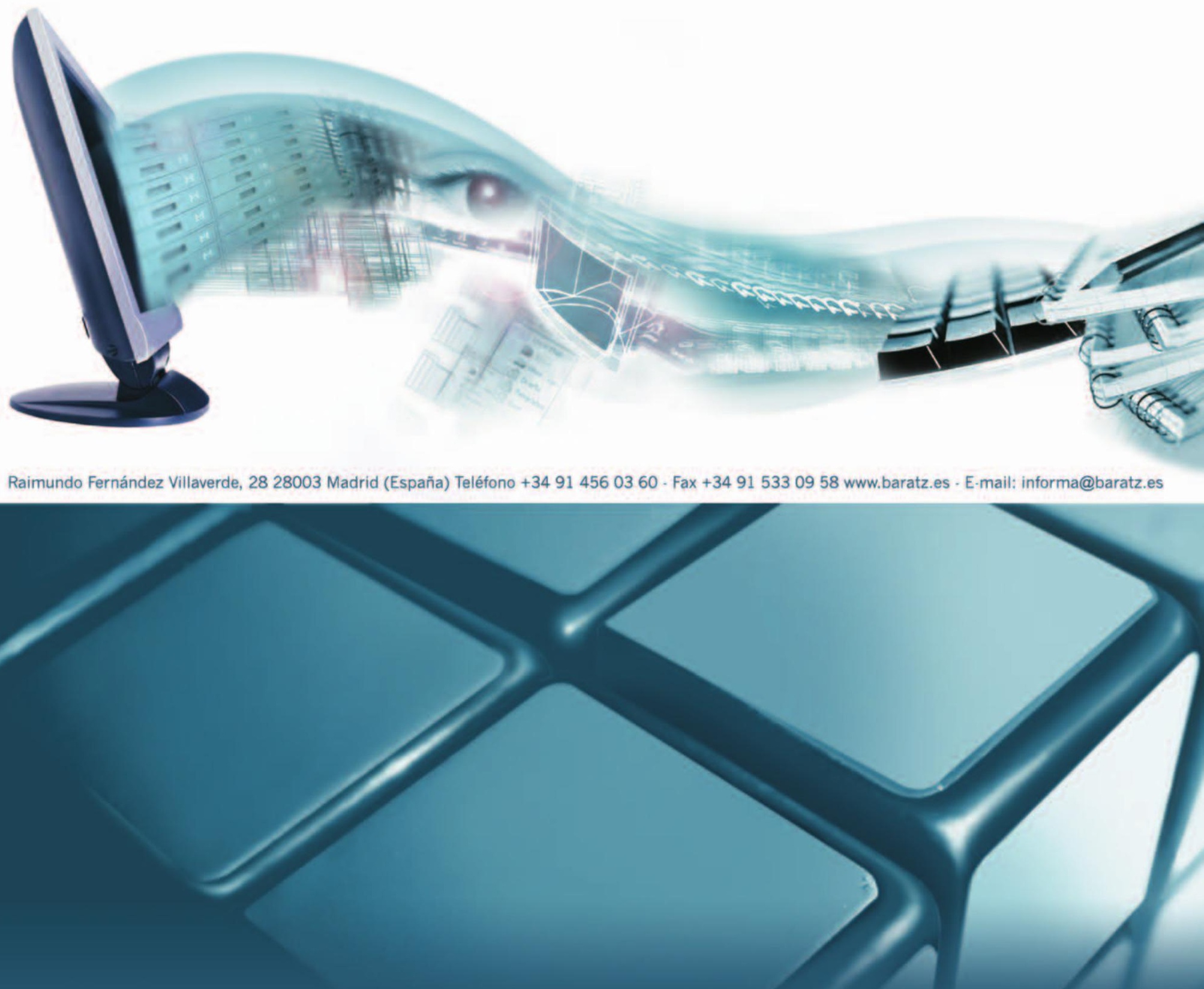

(2) Solvciones para bibliotecas: Absys, absys NET, Absys express

(2) Gestión documental y del conocimiento: BKM, Baratz Windows, BRSCGI

(2) Soluciones para archivos: Albalá

(2) Servicios de Catalogación Retrospectiva

(9) Edición en CD-Rom

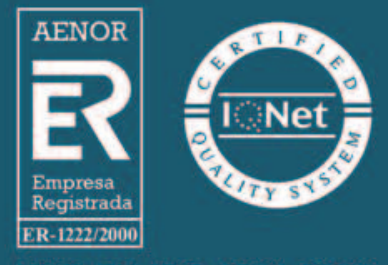


científica, aumentando el dinamismo del sistema científico y enriqueciendo las investigaciones.

Los sistemas actuales de comunicación científica (nos referimos fundamentalmente a las revistas) no parece que puedan ser reemplazados a corto plazo por estos otros sistemas colaborativos. Esto es así debido a la necesidad de garantizar la calidad y veracidad del contenido que se publica en ellas y que se lleva a cabo, generalmente, mediante la evaluación por expertos de los trabajos presentados. Quizá el paso del tiempo ayude a considerar a estas herramientas como mecanismos que enriquezcan las investigaciones y ayuden a garantizar esa calidad y veracidad de contenidos a través de la activa participación de los pares científicos en esta web social.

\section{"Quizá el paso del tiempo ayude a considerar a estas herramientas como mecanismos que enriquezcan las investigaciones"}

Con el fin de encaminarnos hacia la confianza en los contenidos de los blogs, este trabajo presenta una evaluación de aquellos que contienen información de carácter científico, académico o educativo relacionada con la documentación y que son gestionados, generalmente, por organizaciones o profesionales de este ámbito.

El criterio de selección se ha basado en el tipo de contenidos ofrecidos, sin tener en cuenta la procedencia institucional de los autores o responsables. Por este motivo no se han considerado los blogs que aportan, de forma única o mayoritaria posts relacionados con la actividad de una institución (como el caso, por ejemplo, de muchos de los pertenecientes a bibliotecas) o bien aquellos cuyos contenidos se restringen a la temática bibliotecaria y archivística, por considerar que son áreas del conocimiento asimilables en la teoría, pero relativamente alejadas en la práctica profesional; al menos, en lo que se refiere a los contenidos de los blogs, que no suelen mezclar aspectos típicamente "bibliotecarios y archivísticos" (por ejemplo, gestión de colecciones, préstamos, catalogación, etc.), con los típicamente "documentales" (tales como gestión de contenidos, servicios web, bibliometría, etc.). Sirvan de ejemplo los blogs de la biblioteca del Tribunal Superior de Justicia de Andalucía o el de la biblioteca médica del Hospital Universitario de Getafe cuyos contenidos se dedican, como no podría ser de otra forma, a mantener informados a sus usuarios sobre el servicio de la biblioteca y novedades de interés para los mismos. La evaluación se ha basado en el análisis de sus aspectos formales, es decir, qué tipo de contenidos, servicios y adecuación a las formalidades y normas de la publicación web presentan, así como varias propuestas para su mejora.

\section{http://www.lacoctelera.com/infoiudex \\ http://www.infodoctor.org/BiblioGE.php}

\section{2. ¿Es necesario evaluar la calidad formal de un blog?}

En esencia estamos hablando de un portal web más o menos interactivo que publica breves reseñas comentadas y que es actualizado de forma frecuente. Por tanto, al igual que otros contenidos presentes en internet - por ejemplo, las páginas personales o profesionales o los portales de información y de ocio- su calidad formal dependerá de unos criterios comunes a la plataforma sobre la que se difunden: aspectos relativos a su accesibilidad, usabilidad, a los servicios de valor añadido, etc.

No obstante, hemos de recordar que son unas herramientas que poseen, además, características específicas que las diferencian de otros contenidos presentes en la web. Algunas de ellas ayudan a explicar el porqué de la importancia de atender a su calidad formal.

En primer lugar, ofrece la posibilidad de establecer una comunicación de doble sentido con sus usuarios, ya estén registrados o simplemente actúen como invitados anónimos. Aunque cualquier contenido publicado en internet, por definición, exige un nivel de interactividad mayor que el necesario en otros medios, en el caso de los blogs es sin duda todavía superior, precisamente por esa facultad para recibir comentarios. El ejemplo más evidente es el servicio habilitado en la inmensa mayoría de ellos para que los usuarios realicen comentarios a las informaciones publicadas. Si no se ofrece un interfaz limpio, claro e intuitivo, una de las principales ventajas de la herramienta quedará seriamente perjudicada. Es manifiesto, por tanto, el cuidado que se ha de tener respecto al cumplimiento de los criterios de accesibilidad y usabilidad, si se pretende alcanzar la mayor difusión de los contenidos publicados.

Una segunda característica que hace de la evaluación formal de un blog algo imprescindible es su rápida actualización. Debido a que el flujo de información es (o debería ser) casi constante, es conveniente que el sistema posea unas características que hagan fácilmente discernibles los cambios acaecidos desde la anterior consulta. Además, y con la creciente popularización de las tecnologías de agregadores de contenidos, resulta cada vez más urgente que los blogs incorporen al menos alguno de los estándares más conocidos.

Una ventaja que debería traducirse en una mayor exigencia en cuanto al aumento de la calidad reside en que a menudo son herramientas con un alto grado 


\section{"Con la popularización de los agregadores de contenidos, cada vez es más necesario que los blogs incorporen sistemas para conocer sus actualizaciones"}

de homogeneidad entre sí. Es decir, al margen de que se trate de blogs personales, académicos, educativos, institucionales o periodísticos, casi todos ellos comparten una estructura común, e incluso un mismo tipo de contenidos (vínculos a otros, archivos de comentarios, enlaces relacionados, etc.). Además, existen numerosos recursos gratuitos para crearlos de forma rápida y sencilla, con una apariencia correcta y servicios avanzados, lo que favorece esta estandarización.

\section{1. ¿Y los contenidos?}

A pesar de que este aspecto no forma parte de la motivación de nuestro artículo, merece la pena reseñar brevemente algunos problemas relativos a este punto.

Como cualquier otro canal de comunicación, presentan algunos de los problemas inherentes a todo medio de intercambio de información. Uno de ellos, sin duda, es el que hace referencia a la veracidad y calidad de los contenidos que transmite. En el caso de los blogs académicos y científicos este aspecto adquiere especial relevancia, ya que generalmente carecen de los controles de contenido que sí operan en las revistas científicas.

No obstante, antes de proponer un modelo para controlar su calidad conviene reflexionar sobre la naturaleza y objetivos de un blog científico. Actualmente no es un canal sustitutivo de las tradicionales publicaciones científicas, sino una herramienta que agiliza la comunicación informal de la ciencia. Aunque este hecho no implique la dejación de toda responsabilidad en el control de los contenidos, sí aconseja no aplicar controles estrictos con objeto de no lentificar un proceso de comunicación que tiene en la inmediatez, precisamente, una de sus grandes ventajas.

\subsection{Estado de la cuestión}

En la actualidad se han publicado pocos estudios sobre evaluación de calidad formal de blogs, sin que ninguno de ellos haya asumido el estudio desde una perspectiva global. En general, se tratan aspectos más concretos como los contenidos visuales (Scheidt, Wright $^{1}$ ) o la adaptación de los blogs a la web semántica (Karger, Quan ${ }^{2}$ ), que de algún modo pueden relacionarse con aspectos formales. En general, la atención de los investigadores está centrada en una perspectiva que prima el tratamiento documental (clasificación, descripción) de los contenidos (desde el momento en que son entendidos principalmente como sistema gestor de contenidos), o bien en las características sociales que los definen.

Los proveedores de servicios de blogs sí que ha sido objeto de estudios de carácter integrador centrados en la descripción de sus capacidades tecnológicas y las funcionalidades que ofrecen al usuario. El estudio más exhaustivo sobre el tema fue publicado por Westner ${ }^{3}$ en 2004, mientras que Tramullas y Garrido ${ }^{4}$ (2006) han realizado un análisis similar basado, en este caso, en software de carácter gratuito exclusivamente.

\section{Metodología del estudio}

Como ya se ha comentado en la introducción, el objetivo principal es describir las principales características formales que presentan los blogs académicos (se han tenido en cuenta 30) del ámbito de la documentación en España, describiendo sus principales carencias y fortalezas.

La selección se ha realizado teniendo en cuenta los enlaces a otros blogs presentes en cada uno de ellos, debido a que no se dispone de un directorio completo de blogs sobre documentación. Los recursos en español consultados, como Webdirectorio, el buscador de blogs de Google 5 , o populares proveedores de servicios como blogger, no ofrecen datos significativos sobre la cantidad total en nuestro campo. Por otro lado, buscadores especializados como Icerocket, Blogpulse o Waypath tampoco aportan un panorama claro sobre el tema, por lo que se ha considerado que el análisis de los webrolls de cada uno de los recursos más importantes permite detectar las principales y más activas blogs, de acuerdo con la frecuencia en que son enlazadas por otros sitios similares. No obstante, no se ha realizado un muestreo estadístico propiamente dicho, ya que se ha estimado que el número total de blogs académicos españoles y activos no supone una cantidad mucho mayor de los 30 casos estudiados.

\section{http://www.webdirectorio.com \\ http://www.blogger.com \\ http://www.icerocket.com \\ http://www.blogpulse.com \\ http://www.waypath.com}

La evaluación se ha realizado a través de un cuestionario elaborado a partir del modelo propuesto en la Guía de evaluación heurística de sitios web, desarrollada por Hassan y Martín Fernández ${ }^{6}$, y que en forma de checklist permite detectar todos los puntos susceptibles de ser valorados. No obstante, se han efectuado numerosas modificaciones y adiciones al mismo, con objeto de adecuarlo a las necesidades concretas de 


\section{Relación de blogs analizados}

Ec3noticias http://ec3noticias.blogspot.com/

A propósito de http://www_apropositode.blogspot.com/

Catorze http://www.catorze.com/blog/

Baiget info notas http://baiget.blogspot.com/

Biblioblog http://www.interreg-eet.info/weblogs/biblioblog/index.html

Bibliometría http://www.bibliometria.com/

Chacobo http://www.chacobo.com/

Deakialli http://www.deakialli.com/

Documenta2 http://documenta2.blogsome.com/

El documentalista enredado http://www.documentalistaenredado.net/

Documentación http://www.documentacion.com.es/

Documentación informativa http://fvalle.blogspot.com/

Documentación, biblioteconomía e información http://www.lacoctelera.com/documentacion

Documentamanía http://documentamania.blogspot.com/

Documentación sin límites http://docunlimited.blogsome.com/

Métodos de busca http://www.metodosdebusca.com/118/index.php

EcibJaveriana http://ecib.blogspot.com/

El documentalista audaz http://sirmijail.blogspot.com/

Herramienta de documentalista http://www.espacioblog.com/ictineo/

Incatalogable http://www.excatedra.blogspot.com/

Ranganatha2 http://www.espacioblog.com/ranganatha2

Recuperación de información en la web http://irsweb.blogspot.com/

Sedic blog http://blog.sedic.es/

Servicio de alerta http://docdigital.typepad.com/

TAO blog España ByD http://taoblogespanabyd.blogspot.com/

TekaTeka http://tekateka.blogspot.com/

Telendro http://telendro.com.es/

Véase además http://www.veaseademas.com/

Visto y leído http://vistoyleido.blogspot.com/

Web semántica hoy http://www.wshoy.sidar.org/

este estudio, especialmente en lo que se refiere a las características específicas de los blogs. La estructura general de dicho cuestionario, está constituida por cinco grandes bloques:

- Parámetros generales: donde se definen aspectos globales relativos al diseño, estructura, identidad, url y objetivos.

- Identidad, información y servicios: en la que se abarca todo lo referente a la presencia de contenidos e identificación de autores y responsables, así como servicios de ayuda y búsqueda de información.

- Estructuras y navegación: fundamentalmente, haciendo referencia a aspectos relativos a usabilidad.

- Lay-out de la página: que tiene que ver, del mismo modo, con la usabilidad y su aspecto general.

- Accesibilidad: en el que se incluyen las validaciones de css, html/Xhtml, y se aplica el Test de Accesibilidad Web (TAW).

Además, se ha evaluado la visibilidad hipertextual (número de enlaces que cada blog recibe de terceros) según los motores de búsqueda de Google, Yahoo y Microsoft MSN, así como la calidad de los enlaces a través del Page Rank de Google.

La evaluación se ha desarrollado de manera independiente por cada uno de los autores, para después realizar una nueva valoración, esta vez de forma conjunta y consensuada.

\section{Resultados}

\subsection{Parámetros generales}

Teniendo en cuenta la muestra de nuestro estudio, son pocos los blogs mantenidos por instituciones (16\%), siendo mayoría los gestionados por profesionales a título individual.

En un 60\% de los casos se indican claramente los objetivos de forma concreta y bien definida. Este dato indica que en un porcentaje elevado de blogs (40\%) no es posible conocer de manera inmediata cuáles son sus temas de interés, hecho que puede causar cierto desconcierto en los usuarios y una pérdida de lectores potenciales. 


\section{Test de evaluación heurístico de blogs}

\section{- Parámetros generales}

Tipología del blog (personal, institucional).

Objetivos: ¿sson concretos y bien definidos?

Si están definidos, ilos contenidos se ajustan a ellos adecuadamente?

¿Existe una política editorial de aceptación de contribuciones?

$\dot{\imath}$ Tiene dominio propio?

¿Tiene una url correcta, clara y fácil de recordar?

¿Muestra de forma precisa y completa qué contenidos o servicios ofrece realmente?

¿La estructura general del blog está orientada al usuario?

¿Es coherente el diseño general del blog?

¿Es reconocible el diseño general del blog?

¿Se actualiza periódicamente?

¿Ofrece algún tipo de suscripción?

\section{- Identidad, información y servicios}

¿Se muestra claramente la identidad del blog a través de todas las páginas?

¿Existe información sobre el creador del blog?

¿Existe un logotipo?

El logotipo, ¿es significativo, identificable y visible?

$\dot{\imath}$ Existe alguna forma de contacto con los responsables del blog?

En los posts:

- ¿se muestra claramente información sobre el autor? - ¿se muestra claramente la fecha de publicación?

-¿ ofrece enlaces permanentes?

¿Se da información sobre número de usuarios registrados e invitados?

¿Existe un calendario de publicación?

$\dot{\partial}^{E x i s t e}$ un archivo donde consultar posts anteriores?

¿Existe un disclaimer o alguna declaración ética?

¿Ofrece enlaces a otros blogs?

¿Ofrece enlaces externos a otros recursos de información?

¿Dispone de un enlace a trackbacks?

¿Presenta un listado de palabras clave para cada post? ¿Ofrece sindicación de contenidos?

¿Está traducido a otros idiomas?

¿Ofrece la visualización a través de otras plataformas (ej. PDA)?

¿Existe algún tipo de control sobre contenidos polémi$\cos$ ?

¿Posee una sección de ayuda?

En enlace a la sección de "Ayuda", ¿está colocado en una zona visible y estándar?

¿Ofrece una vista previa antes de publicar?

¿Existe algún tipo de buscador?

¿Se encuentra fácilmente accesible?

¿Es fácilmente reconocible como tal?

¿Permite la búsqueda avanzada?

¿Contiene un sistema de búsqueda basado en algún tratamiento documental?

¿Muestra los resultados de forma comprensible para el usuario?
¿La caja de texto es lo suficientemente ancha? ¿Asiste al usuario en caso de no poder ofrecer resultados para una consultada dada?

¿Dispone de una ayuda para realizar la búsqueda?

Número medio de comentarios (10 últimos posts).

\section{- Estructuras y navegación}

La estructura de organización y navegación, ¿es la más adecuada?

¿Hay algún sistema de navegación distinto a la navega ción por fechas?

¿Están clasificados temáticamente los post?

Número de clics necesarios para ver los comentarios a los posts.

Número de clics necesarios para hacer comentarios a los posts.

¿Los enlaces son fácilmente reconocibles como tales? ¿la caracterización de los enlaces indica su estado (visitados, activos, etc.)?

¿Existen elementos de navegación que orienten al usuario acerca de dónde está y cómo deshacer su navegación?

¿Existen páginas "huérfanas"?

\section{- Lay-out de la página}

¿Se aprovechan las zonas de alta jerarquía informativa de la página para contenidos de mayor relevancia? ¿Se ha evitado la sobrecarga informativa? ¿Es una interfaz limpia, sin ruido visual? ¿Existen zonas en "blanco" entre los objetos informativos de la página para poder descansar la vista? ¿Se hace un uso correcto del espacio visual de la página?

¿Se utiliza correctamente la jerarquía visual para expresar las relaciones del tipo "parte de" entre los elementos de la página?

\section{- Accesibilidad}

¿El tamaño de fuente se ha definido de forma relativa? ¿El tipo de fuente, efectos tipográficos, ancho de línea y alineación empleados facilitan la lectura?

$\dot{\imath}$ Existe un alto contraste entre el color de fuente y el fondo?

¿Incluyen las imágenes un texto alternativo que describe su contenido?

$\dot{¿}^{E s}$ compatible el sitio web con los diferentes navegadores?

¿Se visualiza correctamente con diferentes resoluciones de pantalla?

¿Se puede imprimir la página sin problemas?

Evaluación a través de TAW.

Evaluación a través de validador html/Xhtml.

Evaluación a través de validador css.

\section{- Visibilidad}

Link: Google.

Link: Yahoo.

Link: MSN.

PageRank. 
Salvo en casos aislados, no existe una política de aceptación de contribuciones, fundamentalmente porque son los propios gestores los que realizan las aportaciones, aunque los comentarios suelen estar abiertos a la participación de todos los interesados.

En un $70 \%$ no se tiene un dominio propio por lo que, habitualmente, no poseen url fáciles de recordar. Esta situación puede ser debida a que en su mayoría se han elaborado gracias a servicios estándar que permiten crear y publicar blogs de manera sencilla, por no mencionar que los alojan en sus propios servidores.

La práctica totalidad de los blogs analizados muestra una estructura coherente, reconocible y orientada al usuario. Como se indica en el párrafo anterior, los servicios gratuitos de creación y publicación de blogs contribuyen a esta uniformidad, que en cualquier caso puede considerarse muy positiva.

Se ha detectado que en un $24 \%$ no se realizan actualizaciones periódicas, base fundamental de un sistema de comunicación de este tipo, lo que supone una valoración negativa muy relevante.

\subsection{Identidad, información y servicios}

Un aspecto polémico en cuanto a las contribuciones en los blogs es la existencia de algún tipo de control o limitación sobre las aportaciones realizadas por los usuarios. El hecho de trabajar con un software colaborativo implica evitar la aplicación de excesivas restricciones en la participación; sin embargo, cuando se profesionalizan estas herramientas, como es el caso de los blogs analizados en este trabajo, puede surgir la necesidad de establecer ciertas reglas o normas a la colaboración con el fin de evitar intrusiones que afecten a la calidad de los contenidos aportados.

\section{"Se ha detectado únicamente un caso en el que se incluye una declaración ética sobre los contenidos"}

Al analizar la existencia o no de una declaración ética referente a los contenidos, se ha detectado únicamente un caso en el que se incluya esta información. En esta misma línea se ha tratado el tema de control de contenidos; es decir, si antes de que se publique cualquier información ésta pasa por un filtro que evite la publicación de contenidos polémicos. Este tipo de control se ha encontrado solamente en una tercera parte $(37 \%)$.

La sindicación es un servicio de valor añadido muy interesante para los usuarios. Permite estar al tanto de las novedades de una serie de blogs de interés para los lectores habituales, ahorrándoles tiempo y proporcionando información pertinente no sólo por su contenido sino por su actualidad. Tan solo en un 53\% de los blogs se ha identificado algún servicio de este tipo, generalmente rss o $A_{t o m}$, y muy ocasionalmente ambos a la vez.

Por último, hay que destacar dentro de este apartado, que únicamente en un $40 \%$ de los casos se ofrece una búsqueda avanzada, y son todavía menos aquellos blogs que incorporan algún tipo de tratamiento documental a sus entradas.

\subsection{Estructuras y navegación}

Los aspectos relacionados con la navegación suelen estar bien cuidados. Los posts siempre son fácilmente visibles, de manera inmediata, y el acceso al formulario para realizar comentarios se realiza de forma rápida e intuitiva.

No obstante, existe un aspecto que apenas cumple un $7 \%$ de los blogs. Se trata de la existencia de elementos de navegación que orienten al usuario sobre dónde está y como deshacer su navegación. El ejemplo más conocido son los llamados "caminos de migas" (breadcrumb trails), que apenas están presentes. Por lo demás, es una cuestión que no presenta especiales problemas en los casos analizados.

\subsection{Lay-out de la página}

Con motivo de que muchos de los blogs analizados son creados con herramientas automáticas se observan algunos problemas comunes en muchos de ellos. Un ejemplo es el poco aprovechamiento que se realiza de las zonas de alta jerarquía informativa de la página. Esta situación se encontró en un $83 \%$, donde el acceso al buscador, enlaces y secciones se reparten en los menús laterales de las páginas, ocasionando algunos problemas de usabilidad cuando la cantidad de recursos es demasiado grande.

\subsection{Accesibilidad}

Los principales problemas encontrados están relacionados con los siguientes aspectos:

- Cumplimiento de los niveles de accesibilidad: únicamente el 56\% supera el nivel A según el $T A W^{8}$, desarrollado por la Fundación CTIC. Ninguno alcanza el nivel AA (obligatorio, por ejemplo, para las páginas web desarrolladas en el ámbito de la administración pública).

- Ausencia de textos alternativos en las imágenes: solo un $17 \%$ de los casos incorporan las etiquetas correspondientes.

- Superación de los test de validación de $\mathrm{Xhtml}^{9}$ y $\operatorname{css}^{10}$ : solo son superados por un $7 \%$ y un $27 \%$ respec- 


\begin{tabular}{|c|c|c|c|c|c|}
\hline \multicolumn{2}{|c|}{$\begin{array}{l}\text { Porcentaje de criterios de calidad } \\
\text { formal cumplidos por los blogs }\end{array}$} & \multicolumn{4}{|c|}{$\begin{array}{c}\text { Visibilidad hipertextual } \\
\text { y Page Rank }\end{array}$} \\
\hline Criterios relativos a... & $\%$ cumplido & \multirow{2}{*}{$\begin{array}{l}\text { Enlaces } \\
\text { recibidos en }\end{array}$} & \multirow{2}{*}{$\begin{array}{l}\text { Valor } \\
\text { máximo }\end{array}$} & \multirow{2}{*}{$\begin{array}{l}\text { Valor } \\
\text { mínimo }\end{array}$} & \multirow{2}{*}{ Media } \\
\hline Parámetros generales & 64,44 & & & & \\
\hline Identidad e información & 52,63 & Google & 2.040 & 0 & 712,35 \\
\hline Estructura y navegación & 57,04 & Yahoo & 37.200 & 40 & $7.707,90$ \\
\hline Lay-out de la página & 78,89 & & & & \\
\hline Accesibilidad & 55,71 & MSN & 7.030 & 6 & $1.717,55$ \\
\hline Resultado global & 57,21 & Pager Rank & 6 & 3 & 4,43 \\
\hline
\end{tabular}

tivamente. Los servicios de validación empleados han sido desarrollados por el $W 3 C$, y están disponibles de forma gratuita.

\subsection{Visibilidad}

Ha sido analizada a través del número de enlaces recibidos por los blogs (visibilidad hipertextual) y a través del PageRank de Google. La tendencia general es que aquellos blogs que obtienen una mejor puntuación total en el test de análisis reciben un mayor número de enlaces externos, y tienen un PageRank que oscila entre 5 y 6 , que son valores superiores a la media.

\section{Conclusiones y propuesta de mejora}

Basándonos en los resultados presentados anteriormente podemos concluir cuáles son los aspectos mejor integrados en los blogs analizados y los que merecen ser mejor tratados con objeto de mejorar su calidad formal.

\subsection{Puntos fuertes}

En general, se ha observado que presentan una estructura coherente y reconocible a lo largo de todas las páginas, y que los contenidos de los mismos se suelen ajustar a los objetivos que pretenden alcanzar. Prácticamente todos ellos incluyen los contenidos básicos que ha de tener todo blog, a saber: un archivo donde consultar los mensajes anteriores, enlaces a otros (blogroll) y, además, vínculos a diferentes recursos de información relacionados.

La navegabilidad tampoco presenta especiales problemas: muchos de los recursos analizados disponen de sistemas alternativos a la navegación que el recorrido por fechas, y apenas se han encontrado páginas huérfanas en sus sitios web. Los espacios visuales están correctamente organizados, evitando sobrecargas informativas y ofreciendo, por lo general, un aspecto agradable para la lectura.

Además, en la inmensa mayoría de los casos no presentan problemas de legibilidad, pueden ser consultados en diferentes tipos de navegadores sin por ello perder su aspecto original, y no presentan problemas cuando se trata de imprimir sus contenidos.

\subsection{Puntos débiles}

Los principales problemas detectados tienen que ver con la accesibilidad, la sindicación de contenidos y algunos elementos de estructura y navegación.

Sin lugar a dudas, es la accesibilidad el aspecto menos cuidado: son muy pocos los blogs que añaden textos alternativos a imágenes, y son mayoría los que no poseen html ni css considerados válidos según el W3C. Además, ninguno de los casos analizados superó el nivel 2 del TAW.

En cuanto a la sindicación de contenidos, todavía no es una práctica asumida por la mayoría de los responsables. Se trata sin duda de un aspecto cada vez más valorado por el enorme ahorro de tiempo que supone, y que aún queda por incorporar en los blogs españoles.

Respecto a los elementos de estructura y navegación, lo más preocupante es la ausencia de elementos de navegación de apoyo al usuario (como las ya mencionadas breadcrumb trails) y el escaso uso de ciertas partes de alta jerarquía informativa de la página, especialmente la parte superior de la misma.

Otros problemas comunes a muchos blogs son la ausencia de una declaración ética sobre sus contenidos, la escasa actualización periódica y la falta de una definición clara de los objetivos que persiguen.

\subsection{Propuesta de mejora}

Con objeto de contribuir a la optimización de su calidad formal, hemos concretado una serie de recomendaciones que se encuentran agrupadas de manera similar a la utilizada en el cuestionario de evaluación, con objeto de facilitar su comprensión:

- Parámetros generales: resulta esencial que los 
objetivos queden definidos de forma amplia y concreta, que sus contenidos se ajusten a ellos, y que se actualicen al menos semanalmente.

- Identidad e información: los artículos publicados deben estar datados e identificados correctamente. En cuanto a los archivos, deben estar al menos organizados cronológicamente y temáticamente; son imprescindibles y resulta muy recomendable ofrecer un buscador que facilite al usuario ayuda para la recuperación de los resultados. Elementos fundamentales son la presencia de url permanentes para facilitar el enlace desde sitios externos, la adición de trackbacks y la sindicación de los contenidos utilizando alguno de los estándares más populares, y facilitando acceso a las páginas donde es posible descargar los agregadores. Además, resulta conveniente ofrecer al usuario una vista previa antes de publicar los comentarios, como medida de mejora de la calidad.

- Usabilidad: en lo posible, se ofrecerá al usuario alguna alternativa a la navegación por fechas (a través de categorías temáticas, por ejemplo). El acceso a los comentarios deberá ser fácilmente localizable y rápidamente accesible. Además, las zonas superior y central de la página deben de ser aprovechadas para incluir la información más relevante.

- Accesibilidad: la página deberá ofrecer texto a tamaño relativo, incluir etiquetas para la descripción de imágenes y haber superado al menos el primer nivel de alguno de los tests de accesibilidad existentes. Además, conviene validar las hojas de estilo y el código html/ Xhtml en las direcciones oficiales del W3C. Un último aspecto, muy importante, es la adaptación de la página a diferentes navegadores, en especial a Internet Explorer y Firefox, lo que asegura su visibilidad en muchos otros que suelen compartir parámetros similares a los mencionados anteriormente.

La asunción de estas especificaciones no implica, desde luego, una mayor visibilidad o el aumento de la popularidad del blog, pero permiten hacer más sólida la estructura formal sobre la que se apoyan los contenidos incluidos, que son, en última instancia, el criterio decisivo a la hora de determinar su éxito en cuestiones de popularidad.

\section{Notas}

1. Scheidt, L. A.; Wright, E. Common visual design elements of weblogs. Consultado en: 07-11-06.

http://blog.lib.umn.edu/blogosphere/common_visual.html

2. Karger, D. R.; Quan, D. What would it mean to blog on the semantic web?. Consultado en: 07-11-06.

http://theory.csail.mit.edu/ dquan/iswc2004-blog.pdf

3. Westner, Markus K. Weblog service providing. Identification of functional requirements and evaluation of existing weblog services in German and English languages. Consultado en: 07-11-06.

http://westner.levrang.de/cms/upload/pdf/master-dissertation-weblog-blogblogg-weblogs-blogs.pdf

4. Tramullas Saz, J.; Garrido, P. Weblogs content classification tools: performance evaluation. En: Guerrero-Bote, Vicente P. (ed.). Current research in information sciences and technologies. Mérida: Inscit, 2006.

5. Búsqueda de blogs de Google. Consultado en: 07-11-06. http://www.google.com/blogsearch?hl=es

6. Hassan Montero, Y.; Martín Fernández, F. J. Guía de evaluación heurística de sitios web. Consultado en: 05-10-06.

http://www.nosolousabilidad.com/articulos/heuristica.htm

7. Rss 2.0 and Atom 1.0 Compared. Consultado en: 05-10-06. http://www.intertwingly.net/wiki/pie/Rss20AndAtom10Compared

8. Test de Accesibilidad Web. Consultado en: 05-10-06. http://www.tawdis.net

9. W3C Markup Validation Service. Consultado en: 05-10-06 http://validator.w3.org/

10. Servicio de validación de CSS. Consultado en: 05-10-06. http://jigsaw.w3.org/css-validator/

Sonia Jiménez Hidalgo; Javier Salvador Bruna, Centro de Información y Documentación Científica (Cindoc), Consejo Superior de Investigaciones Científicas (Csic).

soniaj@cindoc.csic.es

salvador@cindoc.csic.es

\section{Reducción del embargo online de EPI}

EPI está bajando a 1 año el tiempo de embargo (time embargo, time wall) de acceso de pago a la versión online de la revista. En consecuencia se han empezado a liberar los contenidos del periodo enero 2000-enero 2006, que paulatinamente estarán en acceso abierto en la web de la propia revista: http://www.elprofesionaldelainformacion.com El periodo 1992-1999 ya está disponible en abierto (en html) desde hace meses. 